\title{
Suicidio de médicos. Una realidad ignorada
}

\author{
Suicide of physicians. \\ An ignored reality
}

\author{
Jorge-L Alvarado-Socarras ${ }^{1}$; Edgar-F Manrique-Hernández ${ }^{2}$
}

Forma de citar: Alvarado-Socarras JL, Manrique-Hernández EF. Suicidio de médicos. Una realidad ignorada. Rev Univ Ind Santander Salud. 2019; 51(3): 194-196. doi: http://dx.doi.org/10.18273/revsal.v51n3-2019001 @c) (i)

Desde hace algunos años, a nivel mundial se ha venido registrando un aumento de casos de suicidio entre médicos, desde estudiantes de medicina (pre y post grado) hasta especialistas en diferentes áreas. Tópico que ha generado preocupación en el país, por los recientes casos de suicidios en médicos. Problema aparentemente nuevo, sin embargo, esta situación ya es conocida desde $1900^{1}$. Inicialmente el suicidio en médicos fue considerado como una condición individual, teniendo como solución limitar el ingreso de personas con antecedentes de enfermedades psiquiátricas a la profesión ${ }^{1,2}$. Hoy seguramente persisten profesionales con factores de riesgo de salud mental, pero además con cambios relevantes en la formación médica, ejercicio y retos profesionales, que puedan aumentar el riesgo de compromiso de salud psicobiológica. Pero lo más grave, sin filtros de detección e intervenciones sobre los mismos. A pesar de que en el 2005 la Asociación Americana de Psiquiatría y otras asociaciones recomendaron estudios del problema, para buscar fuertes recomendaciones, estas sugerencias al parecer son ignoradas en la actualidad". Sin embargo, diferentes autores comienzan a describir esto como un problema relacionado con el "ser médico" y encuentran que este problema es multifactorial y no individual como se planteó inicialmente.

Hoy se conoce que la tasa de suicidio entre médicos es más elevada que en la población general, en Estados Unidos una revisión halló que la tasa de suicidio en médicos es de 28 a 40 por 100.000, mayor a la presentada en población general de 12,3 por cada $100.000^{3}$. Schernhammer, et al. encontraron que el Riesgo Relativo (RR) para suicidio en los hombres médicos era 1,41 (IC del 95\% 1,21 a 1,65) y 2,27 (IC de 95\%=1,90 a 2,73) para las mujeres médicas, comparado con la población general ${ }^{4}$. Situación que puede estar subestimada, dado a las malas codificaciones al momento de los registros, por ejemplo, reportando como muerte por intoxicación los casos de suicidio por sobredosis, esto quizás producido por el estigma social que conlleva ${ }^{1,2}$.

Esta situación puede estar relacionada con mayor prevalencia de enfermedades psiquiátricas como depresión, ansiedad e ideas suicidas en comparación con la población general ${ }^{5}$. Un meta-análisis realizado por Milner, et al. ${ }^{6}$ analizó el suicidio por ocupación, encontrando que los más afectados son los trabajadores de ocupaciones elementales tales como peones o limpiadores con un Riesgo Relativo (RR 1,84 IC del 95\% 1,46 a 2,33), seguido de los operarios de máquinas y equipos de cubierta de barco $(\mathrm{RR}=1,78 \mathrm{IC}$ del $95 \% 1,22$ a 2,60) y trabajadores agrícolas $(\mathrm{RR}=1,64$ IC del 95\% 1,19 a 2,28), siendo los trabajadores con menor nivel de habilidades los más afectados comparados con los que se clasificaron como con mayor nivel de habilidades, entre estos los profesionales ${ }^{6}$; sin embargo, a nivel de los médicos, esta condición parece no aplicar. Si bien a nivel ocupacional encontramos otras profesiones afectadas en mayor medida, cabe resaltar que los médicos tienen mayor acceso a métodos letales tales como medicamentos siendo más propensos a sobredosis ${ }^{6}$.

Algunas situaciones pueden ser muy preocupantes, como el inicio de los problemas. Un meta-análisis reciente, estima que la prevalencia de depresión oscila entre 27 y $29 \%$, entre estudiantes de medicina y residente respectivamente, pero puede ser tan alta como del $60 \%$ entre médicos en ejercicio de su profesión ${ }^{5}$. Condición relacionada fuertemente con suicidio. Sin embargo, muy probablemente puede existir un subregistro del diagnóstico ocasionado por falta de

1. Fundación Cardiovascular de Colombia. Bucaramanga, Santander.

2. Escuela Editorial, Salud UIS. Bucaramanga, Colombia.

Correspondencia: Edgar F.Manrique-Hernández. Dirección: Carrera 32 No 29-31 Facultad de Salud UIS. Correo eléctrónico: fabianmh1993@, gmail.com. Teléfono: +3209560967. 
búsqueda de ayuda, pobre introspección, estigmatización social o automedicación de los médicos, planteando serios interrogantes del mayor nivel de estrés al momento de comenzar a ejercer la profesión.

Lo anterior, sumado a otros factores estresantes como largas jornadas laborales, múltiples empleos, fatiga, alto nivel de responsabilidad, temor de cometer errores, condiciones de salud crónica, disminución del autocuidado con rasgos de la profesión médica, que pueden contribuir al estrés profesional ${ }^{2}$. Además, se ha asociado a factores de riesgo como sexo femenino, pertenecer a algunas especialidades como psiquiatría o anestesiología, profesionales jóvenes, solteros y pobre soporte familiar ${ }^{1,7}$.

Pero hay un punto relevante, la depresión, la cual tiene un impacto negativo. Hoy es considerada como la alteración más frecuente del estado de ánimo, con predominio en el sexo femenino, y con un mayor pico entre los 18 y 44 años, guardando relación con suicidio al ajustarse por edad y sexo ${ }^{1}$. En este sentido, entre un 10 a $15 \%$ de las personas que tienen depresión grave se suicidan o al menos lo intentan ${ }^{8}$. Sumado a los datos anotados, la auto prescripción de medicamentos ${ }^{1}$ y la no búsqueda de ayuda profesional hace más serio el problema incluyendo el gremio de psiquiatría, quienes al parecer lideran las tasas de suicidio ${ }^{1,7}$. El suicidio en médicos puede estar precedido de alteraciones mentales entre el 85 al 90\% de los casos, donde el antecedente de depresión puede jugar un rol importante, además, detonantes como consumo de alcohol, abuso sustancias, historia familiar de alteraciones psiquiátricas, médicos huérfanos, dificultades en el hogar, problemas económicos, estado mental actual por ejemplo ansiedad, obesidad, síndrome de fatiga crónica son otros factores relacionados ${ }^{9}$.

Es una condición evidentemente multifactorial, con manifestaciones desde los primeros años de formación, ocasionado por el estrés académico, altas expectativas sociales, familiares y personales, competitividad, generando un aumento de los pensamientos suicidas durante el primer año de formación ${ }^{1,10}$, llegando a presentar estas ideas hasta en un $17,4 \%$ de los estudiantes de medicina ${ }^{11}$. Estas condiciones estresantes aumentan durante la progresión de la carrera médica, por ejemplo, durante el servicio social obligatorio se adquiere una gran responsabilidad, enfrentándose a las dificultades propias del sistema, depresión secundaria a disminución de su interacción con su red de apoyo y ansiedad en el ejercicio profesional principalmente. En el ámbito laboral resulta estresante desempeñar esta labor por el alto componente emocional que denota el desarrollo de la medicina, tipos de personalidad principalmente narcisistas, compulsivos y competitivos de sus profesionales, estrés propio de la actividad clínica, acoso y degaste profesional, así como, discusiones con colegas y otros profesionales de la salud, también sometidos a este estrés ${ }^{1,2}$. No menos importante, es observar una sociedad médica frustrada e insatisfecha de su práctica profesional, con demandas cada vez más severas por mala praxis, ejerciendo una medicina de "miedo" más que de gusto y amor; jugando un rol fundamental en la ideación suicida y suicidio consumado entre el personal de medicina, con un impacto negativo económico en los sistemas de salud. Esta situación obliga a replantear el origen de estos problemas, que pueden iniciar en la selección de los estudiantes de pregrado, con una equivocada orientación curricular y fallas en las competencias interpersonales, entre otras ${ }^{12}$.

Si bien los factores mencionados, son históricos como factores de alteración de salud mental. Los cambios generados en los servicios de salud, como eficiencia, costo efectividad, calidad asistencial y seguridad de usuarios, son determinantes muy valorados en el financiamiento de los proyectos de salud y pueden jugar un nuevo rol en alteraciones del estado mental entre los médicos; disminuyendo la satisfacción laboral, llevando a mayor incidencia de burnout y depresión ${ }^{13}$ ocasionado por la sobrecarga laboral, presión en el ejercicio profesional, aumento de las peticiones y solicitudes por parte de los pacientes, y sobrerregulación de las instituciones. Requiriendo que estas intervenciones incluyan dentro de sus objetivos el adecuado bienestar y salud de los médicos para su correcto funcionamiento.

Sumado a estas causas, las estrategias y búsqueda de ayuda resulta difícil entre los profesionales de medicina, pues primero se debe asumir el rol de enfermo, lo cual conlleva a una dificultad para reconocer ante sus colegas estos problemas, a su vez, la dificultad para la toma de recursos disponibles por vergüenza o miedo a ser estigmatizados ${ }^{2,10}$. Es por ello, que para prevenir el suicidio se deben iniciar intervenciones desde la formación en las universidades, reconociendo los problemas, enseñando pautas de autocuidado, detección de signos de ansiedad, depresión e ideación suicida propias y de sus colegas e intervenciones a realizar, confidencialidad en la toma de programas de alivio, apoyo laboral, disminución de la sobrecarga laboral, mejoras económicas, entre otras ${ }^{1,2,10}$. 
A pesar del panorama oscuro y complejo, programas de intervención temprano abordados desde las escuelas de medicina, dirigidos a la salud mental, pueden disminuir los síntomas depresivos e ideas suicidas ${ }^{14}$. Finalmente, para disminuir las incidencias del suicidio en los médicos, se requieren intervenciones multidisciplinarias, que permitan detectar de forma precoz las alteraciones de salud mental y creación de políticas de intervención.

\section{Referencias}

1. Eckleberry-Hunt J, Lick D. Physician depression and suicide: a shared responsibility. Teach Learn Med. 2015; 27(3): 341-345 doi: https://doi.org/10.1080/10401334.2015.1044751.

2. Mingote Adán JC, Crespo Hervás D, Hernández Álvarez M, Navío M, García-Pando CR. Prevención del suicidio en médicos. Med Segur Trab. 2013; 59(231): 176-204.

3. T’Sarumi O. Physician suicide: a silent epidemic. Reunión Anual de la American Psychiatric Association (APA). Nueva York, Estados Unidos. 2018; 1-227.

4. Schernhammer ES, Colditz GA. Suicide rates among physicians: a quantitative and gender assessment (metaanalysis). Am J Psychiatry. 2004; 161(12): 2295-2302.

5. Petrie K, Crawford J, Baker STE, Dean K, Robinson J, Veness BG. Interventions to reduce symptoms of common mental disorders and suicidal ideation in physicians: a systematic review and meta-analysis. Lancet Psychiatry. 2019; 6(3): 225-234. doi: https://doi.org/10.1016/S2215-0366(18)30509-1.

6. Milner A, Spittal MJ, Pirkis J, LaMontagne AD. Suicide by occupation: systematic review and meta-analysis. $\mathrm{Br}$ J Psychiatry. 2013; 203(6): 409-416. doi: https://doi.org/10.1192/bjp.bp.113.128405.

7. Bailey E, Robinson J, McGorry P. Depression and suicide among medical practitioners in Australia. Intern Med J. 2018; 48(3): 254-258. doi: https://doi.org/10.1111/imj.13717.

8. González Escobar S, Valdez Medina JL. Significado psicológico de la depresión en médicos y psicólogos. Psicol Salud. 2005; 15(2): 257-262.

9. Sheikhmoonesi F, Zarghami M. Prevention of physicians' suicide. Iran J Psychiatry Behav Sci. 2014; 8(2): 1-3.

10. Rotenstein LS, Ramos MA, Torre M, Segal JB, Peluso MJ, Guille C, et al. Prevalence of depression, depressive symptoms, and suicidal ideation among medical students: a systematic review and meta-analysis. JAMA. 2016; 316(21): 2214-2236. doi: https://doi.org/10.1001/jama.2016.17324.

11. Sobowale K, Zhou N, Fan J, Liu N, Sherer R. Depression and suicidal ideation in medical students in China: a call for wellness curricula. Int J Med Educ. 2014; 5: 31-36. doi: https://doi.org/10.5116/ijme.52e3.a465.

12. Ramírez-Villaseñor I. La infelicidad de los médicos: Un fenómeno complejo y un intento por comprenderla. Rev Mex Med Fam. 2018; 1: 37-44.

13. Tateno M, Jovanović N, Beezhold J, Uehara-Aoyama K, Umene-Nakano W, Nakamae T, et al. Suicidal ideation and burnout among psychiatric trainees in Japan. Early Interv Psychiatry. 2018; 12(5): 935-937. doi: https://doi.org/10.1111/eip.12466.

14. Thompson D, Goebert D, Takeshita JA Program for reducing depressive symptoms and suicidal ideation in medical students. Acad Med. 2010; 85(10): 1635-1639. doi: https://doi.org/10.1097/ACM.0b013e3181f0b49c. 